\title{
The XLindley Distribution: Properties and Application
}

\author{
Sarra Chouia, Halim Zeghdoudi ${ }^{*}$ \\ LaPS Laboratory, Badji Mokhtar University, Annaba, Algeria
}

\section{ARTICLE INFO}

Article History

Received 19 Mar 2019

Accepted 31 Jan 2021

\section{Keywords}

Exponential distribution

Lindley distribution

quantile function

method of moment

maximum likelihood method

simulation

2000 Mathematics Subject

Classification: 62E15; 62N02,

62N05; 62P10

\section{ABSTRACT}

This paper proposes a new distribution called XLindley distribution (XLD), this distribution is generated as a special mixture of two distributions: exponential and Lindley and hence the name proposed. Also, the statistical properties like stochastic ordering, quantile function, the maximum likelihood method and method of moments. An application of the model to a real data set presented finally and compared with the fit and shows that XLD has more flexibility than others one-parameter distributions.

(C) 2021 The Authors. Published by Atlantis Press B.V. This is an open access article distributed under the CC BY-NC 4.0 license (http://creativecommons.org/licenses/by-nc/4.0/).

\section{INTRODUCTION}

The real-life applications of contemporary numerical techniques in different fields such as medicine, finance, biological engineering sciences and statistics. To this end, statistics plays a critical role in our real-life applications. Often by using the statistical analysis which strongly depends on the assumed probability model or distributions. However, several problems in statistics do not follow any of the classical or standard probability models.

Let $\mathrm{X}$ is a random variable following the one-parameter distribution with density function called Lindley (LD) distribution:

$$
f(x ; \theta)= \begin{cases}\frac{\theta^{2}(1+x) e^{-\theta x}}{1+\theta} & \mathrm{x}, \theta>0 \\ 0 & \text { otherwise }\end{cases}
$$

It has introduced by Lindley [1]. Sankaran [2] used (1) as mixing distribution of poisson parameter which it named poisson-LD distribution. Recently, Asgharzadeh et al. [3], Zeghdoudi and Nedjar [4], Zeghdoudi and Bouchahed [5], Beghriche and Zeghdoudi [6], Ghitany et al. $[7,8$,$] rediscovered and studies the distribution bounded to (1), what that derived is known as Zero-truncated poisson-LD and pareto$ poisson-LD distributions.

Recently, Zeghdoudi and Nedjar $[9,10]$ introduced a new distribution named Gamma LD distribution based on mixture of gamma distribution with scale parameter $\theta$, mixture parameter $\beta$ and shape parameter 2 and LD distribution with parameter $\theta$. This idea about mixture of two known distributions is not new, there are a lot of mathematicians has use it before like Shanker and Sharma [11] to create a two parameter LD distribution.

The idea of this work is based on special mixture of exponential and LD distributions in order to create the XLindley distribution (XLD). This work is motivated by the following: XLD it is simple and easy to apply; the formulas of the mean, variance, coefficient of variation, skewness, kurtosis and index of dispersion are simple in form and may be used as quick approximations in many cases. However, in general it is applicable to try out simpler distributions than more complicated ones; the XLD can be used quite effectively in analyzing many real lifetime data set: application to Ebola, Corona and Nipah virus and gives adequate fits too many data sets. 
The paper is organized as follows: Section 2 is devoted to introduce the methodology and gives survivals properties of XLD. Section 3 discusses the estimation of its parameter using method of moment and maximum likelihood. Finally, we present illustrative example of XLD with other distributions to show the superiority and flexibility of this model that found.

\section{METHODOLOGY AND SURVIVAL PROPERTIES}

In this section, a mixture of two known distributions used to give new distribution called $X L D$. Let $\mathbf{X}$ be a random variable following mixture distribution, it's density function (pdf) $f(x)$ given in this form:

$$
f(x)=\sum_{i=1}^{k} p_{i} f_{i}(x)
$$

With:

- $f_{i}(x)$ probability density function for each i

- $p_{i} \mathrm{i}=1 \ldots . . \mathrm{k}$ denote mixing proportions that are no-negative and $\sum_{i=1}^{k} p_{i}=1$

We consider

$f_{1}(x) \sim \operatorname{Exp}(\theta)$ and $f_{2}(x) \sim \operatorname{LD}(\theta)$ two independents random variables with $p_{1}=\frac{\theta}{1+\theta}$ and $p_{2}=1-\frac{\theta}{1+\theta}$ respectively. Now the density function of $\mathrm{X}$ is given by:

$$
f_{X L}(x ; \theta)= \begin{cases}\frac{\theta^{2}(2+\theta+x) e^{-\theta x}}{(1+\theta)^{2}} & \mathrm{x}, \theta>0 \\ 0 & \text { otherwise }\end{cases}
$$

The first derivative of $f_{X L}$ is:

$$
\frac{d f_{X L}(\mathrm{x})}{d x}=\frac{-\theta^{2}\left[\theta^{2}+\theta(2+x)-1\right]}{(1+\theta)^{2}} e^{-\theta x}=0
$$

gives:

$$
x=\frac{-\left(\theta^{2}+2 \theta-1\right)}{\theta}
$$

For:

1. $0 \prec \theta \prec \sqrt{2}-1: \hat{\mathrm{x}}=-\frac{\left(\theta^{2}+2 \theta-1\right)}{\theta}$ is critical point which $f_{X L}(\stackrel{\wedge}{\mathrm{x}} ; \theta)$ is maximum

2. $\theta \geq \sqrt{2}-1: \frac{d}{d x} f_{X L}(x ; \theta) \leq 0$, then the density function $f_{X L}(x ; \theta)$ is decreasing in $\mathrm{x}$

And the second derivative is:

$$
\frac{d^{2} f_{X L}(\mathrm{x})}{d x^{2}}=\frac{\theta^{3}\left[\theta^{2}+\theta(2+x)-2\right]}{(1+\theta)^{2}} e^{-\theta x}
$$

Therefore, the mode of XL is given by:

$$
\operatorname{mode}(X)= \begin{cases}-\frac{\left(\theta^{2}+2 \theta-1\right)}{\theta} & \text { for } 0<\theta<\sqrt{2}-1 \\ 0 & \text { otherwise }\end{cases}
$$

We can find easily the cumulative distribution function (CDF) of the XLD:

$$
F_{X L}(x ; \theta)=1-\left(1+\frac{\theta x}{(1+\theta)^{2}}\right) e^{-\theta x} \mathrm{x}>0, \theta>0
$$

The shapes of the PDF, CDF and hazard function of the XLD distribution are given in Figures 1-3 for different values of the parameter theta. 


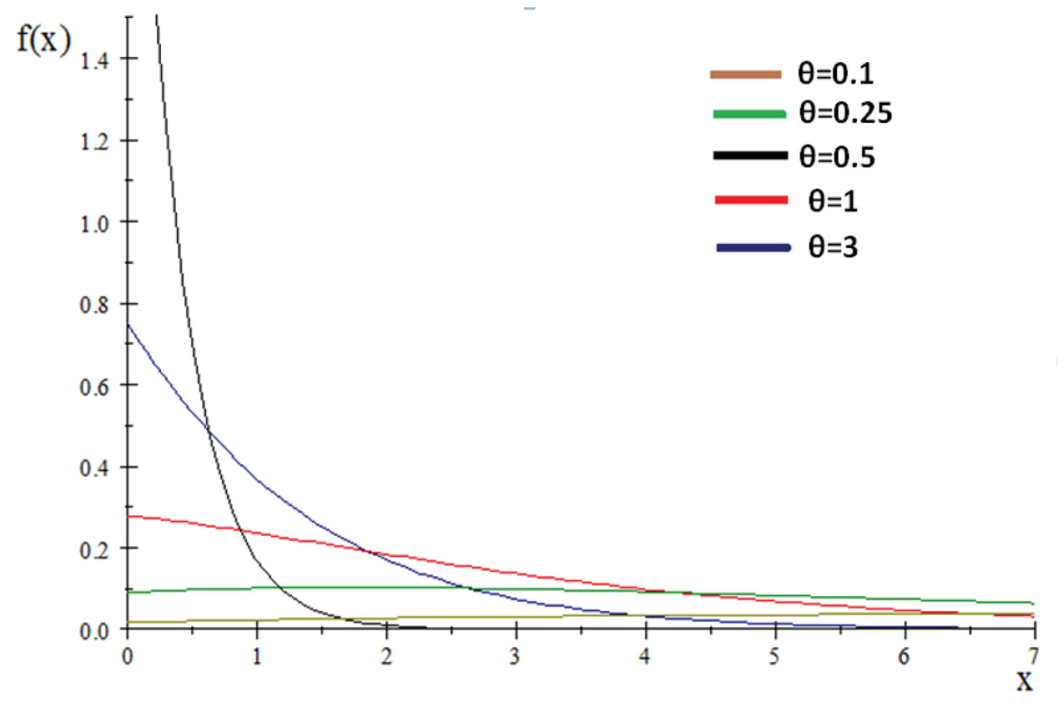

Figure 1 Plots of the density function for some parameter values

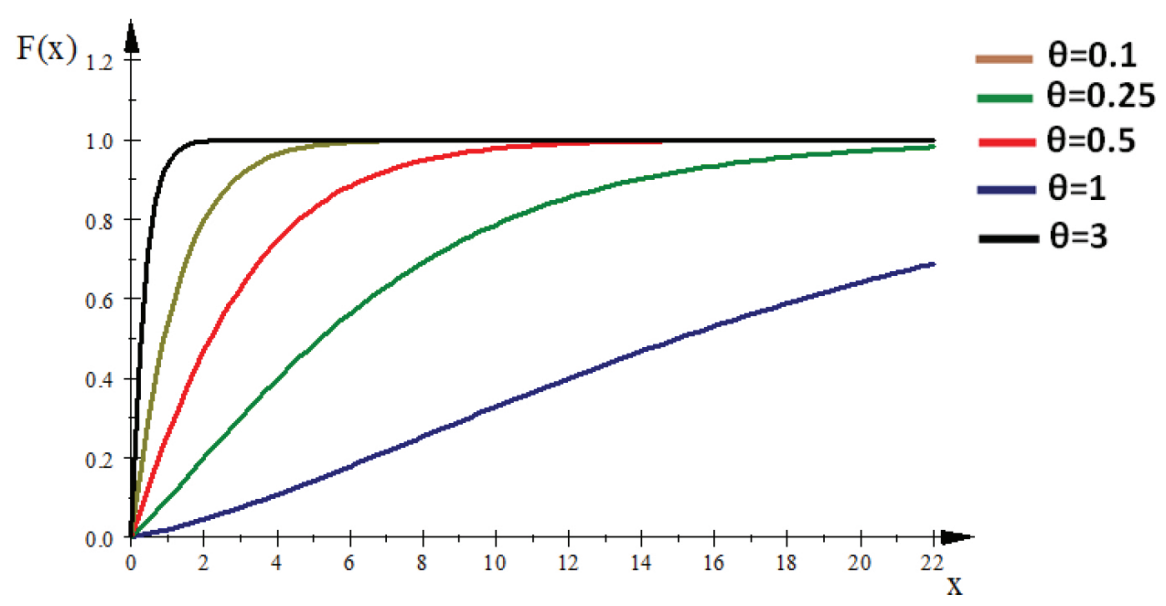

Figure 2 Plots of cumulative distribution function (CDF) for some parameter values

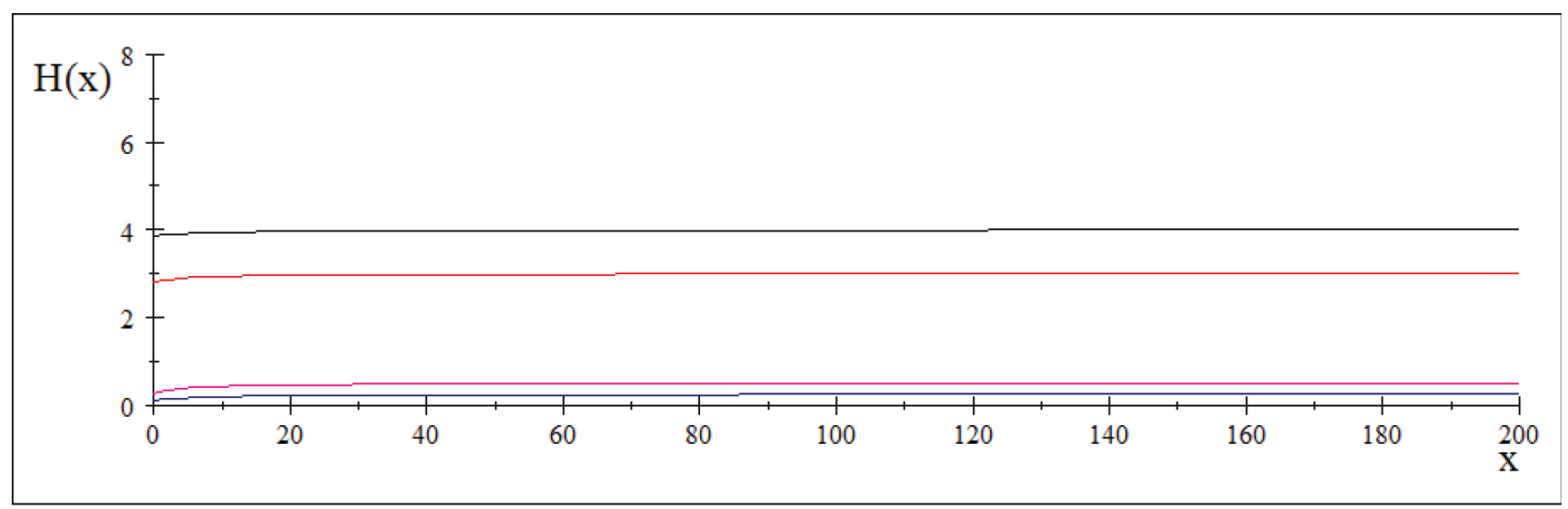

Figure 3 Plots of hazard function for some parameter values: blue (0.25); pink (0.5); red (3); black (4)

\section{SURVIVAL AND HAZARD RATE FUNCTION}

The survival function and failure rate (hazard rate) function for a continuous distribution are defined as: 
Let:

$$
\begin{gathered}
S_{X L}(x)=1-F_{X L}(x) \\
S_{X L}(x)=1-\left[1-\left(1+\frac{\theta x}{(1+\theta)^{2}}\right) e^{-\theta x}\right] \\
S_{X L}(x)=\left(1+\frac{\theta x}{(1+\theta)^{2}}\right) e^{-\theta x}
\end{gathered}
$$

and:

$$
\begin{gathered}
H_{X L}(x)=\frac{f_{X L}(x)}{1-F_{X L}(x)} \\
H_{X L}(x)=\frac{\theta^{2}(x+\theta+2)}{(1+\theta)^{2}\left(x \frac{\theta}{(1+\theta)^{2}}+1\right)} \\
H_{X L}(x)=\frac{\theta^{2}(x+\theta+2)}{(1+\theta)^{2}+x \theta}
\end{gathered}
$$

be the survival and hazard rate function, respectively.

Proposition 1. Let $\boldsymbol{H}_{X L}(\boldsymbol{x})$ be the hazard rate function of $X$. Then $\boldsymbol{H}_{X L}(\boldsymbol{x})$ is increasing.

Proof. According to Glaser [12] and from the density function (2):

$$
\begin{aligned}
\rho(x) & =-\frac{f_{X L}(x)}{f_{X L}(x)}=\frac{x \theta+\theta^{2}+2 \theta-1}{x+\theta+2} \\
& =\frac{1}{x+\theta+2}\left(x \theta+\theta^{2}+2 \theta-1\right)
\end{aligned}
$$

It follows that:

$$
\rho^{\prime}(x)=\frac{1}{(x+\theta+2)^{2}}
$$

Imply that $\mathbf{h}_{\mathrm{XL}}(\mathbf{x})$ is increasing.

\section{MOMENTS AND RELATED MEASURES}

The rth moment about the origin of the XLindey distribution can be obtained as:

$$
\begin{aligned}
\mu_{r}^{\prime} & =E\left(X^{r}\right)=\int_{0}^{\infty} x^{(r)} f_{X L}(x) d x \\
& =\int_{0}^{\infty} x^{(r)} \frac{\theta^{2}(2+\theta+x)}{(1+\theta)^{2}} e^{-\theta x} d x \\
& =\frac{\theta^{2}}{(1+\theta)^{2}} \int_{0}^{\infty} x^{(r)}(2+\theta+x) d x
\end{aligned}
$$

Using gamma integral and little algebraic simplification, we get finally a general expression for the rth factoriel moment of XLD as:

$$
\mu_{r}^{\prime}=\frac{\left(\theta^{2}+2 \theta+r+1\right) r !}{(1+\theta)^{2} \theta^{r}}
$$


Substituting $\mathbf{r}=\mathbf{1 , 2 , 3}$ and $\mathbf{4}$ in (9), the first four moments can be obtained and then using the relationship between moments about origin and moment about mean, the first four moment about origin of XLD were obtained as:

$$
\begin{gathered}
\mu_{1}^{\prime}=\frac{\left(\theta^{2}+2 \theta+2\right)}{(1+\theta)^{2} \theta}=\frac{1}{\theta}+\frac{1}{(1+\theta)^{2} \theta} \\
\mu_{2}^{\prime}=\frac{2\left(\theta^{2}+2 \theta+3\right)}{(1+\theta)^{2} \theta^{2}} \\
\mu_{3}^{\prime}=\frac{6\left(\theta^{2}+2 \theta+4\right)}{(1+\theta)^{2} \theta^{3}} \\
\mu_{4}^{\prime}=\frac{24\left(\theta^{2}+2 \theta+5\right)}{(1+\theta)^{2} \theta^{4}}
\end{gathered}
$$

Proposition 2. Let $X \sim X L(x)$, the mean, variance, coefficients of variation, skewness and kurtosis for $X$ are:

$$
\begin{gathered}
\mu_{1}=E(X)=\frac{(1+\theta)^{2}+1}{(1+\theta)^{2} \theta} \\
E\left(X^{2}\right)=\frac{2\left(\theta^{2}+2 \theta+3\right)}{(1+\theta)^{2} \theta^{2}} \\
\mu_{2}=\operatorname{Var}(X)=\frac{(1+\theta)^{4}+4 \theta^{2}+6 \theta+1}{(1+\theta)^{4} \theta^{2}}
\end{gathered}
$$

Skewness, Kurtosis and Coefficient of variation of XLD:

$$
\begin{gathered}
\text { Skewness }=\sqrt{\beta_{1}}=\frac{E\left(X^{3}\right)}{(\operatorname{Var}(X))^{\frac{3}{2}}}=\frac{6\left(\theta^{2}+2 \theta+4\right)(1+\theta)^{4}}{\left[(1+\theta)^{4}+4 \theta^{2}+6 \theta+1\right]^{\frac{3}{2}}} \\
\text { Kurtosis }=\beta_{2}=\frac{E\left(X^{4}\right)}{(\operatorname{Var}(X))^{2}}=\frac{24\left(\theta^{2}+2 \theta+5\right)(1+\theta)^{6}}{\left[(1+\theta)^{4}+4 \theta^{2}+6 \theta+1\right]^{2}} \\
C . V=\gamma=\frac{\sqrt{\operatorname{Var}(X)}}{E(X)}=\frac{\sqrt{(1+\theta)^{4}+4 \theta^{2}+6 \theta+1}}{(1+\theta)^{2}+1}
\end{gathered}
$$

The coefficients are increasing functions in $\theta$ (see Figure 4 for the graphe of C.V $(\gamma)$ and Skewness $\sqrt{\beta_{1}}$ for varying $\theta$ ).

\section{STOCHASTIC ORDERING}

Definition 1. Consider two random variables $X$ and $Y$. Then $X$ is said to be smaller than $Y$ in the:

a) Stochastic order $\left(X \prec_{S} Y\right)$ if $F_{X}(t)>F_{Y}(t), \forall t$.

b) Convex order $\left(X \prec_{C X} Y\right)$, if for all convex functions $\phi$ and provided expectation exist, $E[\phi(X)] \leq E[\phi(Y)]$.

c) Hazard rate order $\left(X \prec_{h r} Y\right)$, if $h_{X}(t) \geq h_{Y}(t), \forall t$.

d) Likelihood ratio order $\left(X \prec_{l r} Y\right)$, if $\frac{f_{X}(t)}{f_{Y}(t)}$ is decreasing in $t$. 


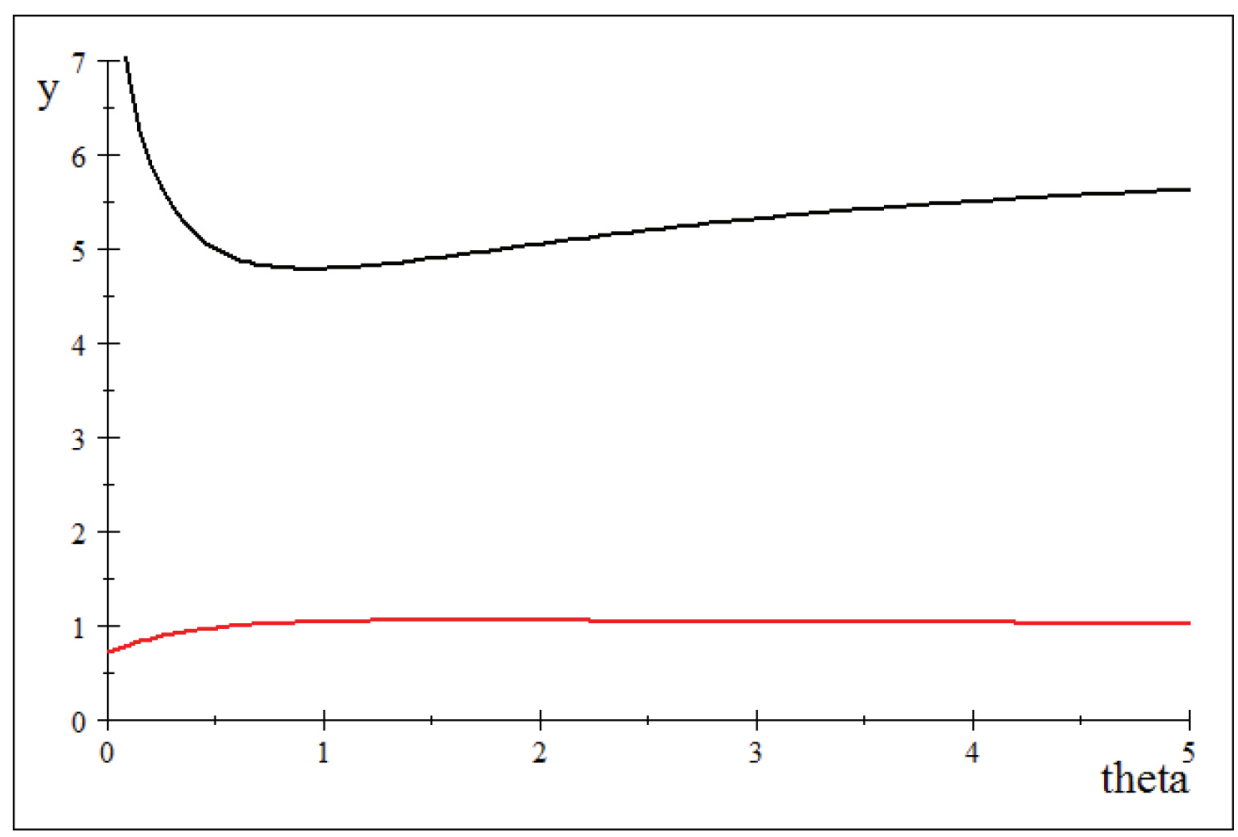

Figure 4 Coefficients for variation (red) and skewness (black)

Remark 1. Likelihood ratio order $\Rightarrow$ Hazard rate order $\Rightarrow$ Stochastic order. If $E(X)=E(Y)$, then: Convex order $\Leftrightarrow$ Stochastic order.

Theorem 1. Let $X_{i} \sim X L\left(\theta_{i}\right), i=1,2$ be two random variables. If $\theta_{1} \geq \theta_{2}$, then $X_{1} \prec_{l r} X_{2}, X_{1} \prec_{h r} X_{2}, X_{1} \prec_{S} X_{2}$.

Proof. We have:

$$
\frac{f_{X_{1}}(t)}{f_{X_{2}}(t)}=\frac{\theta_{1}^{2}\left(2+\theta_{1}+t\right)\left(1+\theta_{2}\right)^{2}}{\theta_{2}^{2}\left(2+\theta_{2}+t\right)\left(1+\theta_{1}\right)^{2}} e^{-\left(\theta_{1}-\theta_{2}\right) t}
$$

For simplification, we use $\ln \left(\frac{f_{X_{1}}(t)}{f_{x_{2}}(t)}\right)$. Now, we can find

$$
\frac{d}{d t} \ln \left(\frac{f_{X_{1}}(t)}{f_{X_{2}}(t)}\right)=-\frac{\theta_{1}-\theta_{2}}{\left(t+\theta_{1}+2\right)\left(t+\theta_{2}+2\right)}-\left(\theta_{1}-\theta_{2}\right)
$$

To this end, if $\theta_{1} \geq \theta_{2}$, we have $\frac{d}{d t} \ln \left(\frac{f_{X_{1}}(t)}{f_{X_{2}}(t)}\right) \leq 0$. This means that $X_{1} \prec_{l r} X_{2}$. Also, according to Remark 1 the theorem is proved.

\section{ESTIMATION OF PARAMETER}

\subsection{Method of Moments Estimation}

Let $\bar{X}$ be the sample mean, equating sample mean and population mean $\mathrm{E}(\mathrm{x})$ :

$$
E(x)=\sum_{i=1}^{n} \frac{x_{i}}{n}
$$

Putting the expression of $\mathrm{E}(\mathrm{x})$ from equation (10) in the equation and solving the equation for $\theta$, We get:

$$
\bar{X}=\frac{(1+\theta)^{2}+1}{(1+\theta)^{2} \theta}=\frac{\theta^{2}+2 \theta+2}{\theta^{3}+2 \theta^{2}+\theta}
$$


We obtain equation of 3rd degree: $\bar{X} \theta^{3}+\theta^{2}(2 \bar{X}-1)+\theta(\bar{X}-2)-2=0$, We take the real part for the solution:

$$
\begin{aligned}
& \hat{\theta}_{M o M}=-\frac{1}{3 \bar{X}}(2 \bar{X}-1)+\frac{\frac{2}{9 \bar{X}}+\frac{1}{9 \bar{X}^{2}}+\frac{1}{9}}{\sqrt[3]{\sqrt{\frac{1}{27 \bar{X}}+\frac{13}{36 \bar{X}^{2}}+\frac{1}{9 \bar{X}^{3}}+\frac{1}{27 \bar{X}^{4}}}+\frac{11}{18 \bar{X}}+\frac{1}{9 \bar{X}^{2}}+\frac{1}{27 \bar{X}^{3}}+\frac{1}{27}}} \\
& +\sqrt[3]{\sqrt{\frac{1}{27 \bar{X}}+\frac{13}{36 \bar{X}^{2}}+\frac{1}{9 \bar{X}^{3}}+\frac{1}{27 \bar{X}^{4}}}+\frac{11}{18 \bar{X}}+\frac{1}{9 \bar{X}^{2}}+\frac{1}{27 \bar{X}^{3}}+\frac{1}{7 \bar{X}}}
\end{aligned}
$$

\subsection{Maximum Likelihood Estimation}

Let $X_{i} \sim X L(\theta), i=1 \ldots \ldots . . n$ be $\mathrm{n}$ random variables. The $\ln$-likelihood function $\ln l\left(x_{i} ; \theta\right)$ is:

$$
L(\theta)=\left(\frac{\theta^{2}}{(1+\theta)^{2}}\right)^{n} \prod_{i=1}^{n}\left(2+\theta+x_{i}\right) e^{-\theta} \sum_{i=1}^{n} x_{i}
$$

Logarithm of likelihood function is:

$$
\begin{aligned}
& \ln l\left(x_{i} ; \theta\right)=2 n \log \theta-2 n \log (1+\theta)+\sum_{i=1}^{n} \log \left(2+\theta+x_{i}\right)-\theta \sum_{i=1}^{n} x_{i} \\
& \ln l\left(x_{i} ; \theta\right)=2 n[\log \theta-\log (1+\theta)]+\sum_{i=1}^{n} \log \left(2+\theta+x_{i}\right)-\theta \sum_{i=1}^{n} x_{i}
\end{aligned}
$$

The derivative of $\ln l\left(x_{i} ; \theta\right)$ with respect to $\theta$ is:

$$
\begin{aligned}
& \frac{d \ln l\left(x_{i} ; \theta\right)}{d \theta}=0 \\
& \frac{d \ln l\left(x_{i} ; \theta\right)}{d \theta}=\frac{2 n}{\theta}-\frac{2 n}{1+\theta}+\sum_{i=1}^{n}\left(\frac{1}{2+\theta+x_{i}}\right)-\sum_{i=1}^{n} x_{i} \\
& \frac{d \ln l\left(x_{i} ; \theta\right)}{d \theta}=\frac{2}{\theta}-\frac{2}{1+\theta}+\frac{1}{n} \sum_{i=1}^{n}\left(\frac{1}{2+\theta+x_{i}}\right)-\bar{X} \\
& \frac{d \ln l\left(x_{i} ; \theta\right)}{d \theta}=\frac{2}{\theta(1+\theta)}+\frac{1}{n} \sum_{i=1}^{n}\left(\frac{1}{2+\theta+x_{i}}\right)-\bar{X}
\end{aligned}
$$

To obtain the maximum likelihood estimation (MLE) of $\theta: \hat{\theta}_{M L E}$ can maximize equation (11) directly with respect to $\theta$, or we can solve the non-linear equation $\frac{d \ln l\left(x_{i} ; \theta\right)}{d \theta}=0$. Note that $\hat{\theta}_{M L E}$ cannot solved analytically; numerical iteration techniques, such as the Newton-Raphson algorithm, are thus adopted to solve the Logarithm of likelihood equation for which (11) is maximized.

The following theorem shows that the estimator of $\theta$ is positively biased.

Theorem.2. the estimator $\hat{\theta}$ of $\theta$ is positively biased, i.e: $E(\hat{\theta})-\theta>0$

Proof. Let $g(\bar{x})=\hat{\theta}$

$$
\begin{aligned}
g(t) & =-\frac{1}{3 t}(2 t-1)+\frac{\frac{2}{9 t}+\frac{1}{9 t^{2}}+\frac{1}{9}}{\sqrt[3]{\sqrt{\frac{1}{27 t}+\frac{13}{36 t^{2}}+\frac{1}{9 t^{3}}+\frac{1}{27 t^{4}}+\frac{11}{18 t}+\frac{1}{9 t^{2}}+\frac{1}{27 t^{3}}+\frac{1}{27}}}} \\
& +\sqrt[3]{\sqrt{\frac{1}{27 t}+\frac{13}{36 t^{2}}+\frac{1}{9 t^{3}}+\frac{1}{27 t^{4}}+\frac{11}{18 t}+\frac{1}{9 t^{2}}+\frac{1}{27 t^{3}}+\frac{1}{7 t}}}
\end{aligned}
$$

and it is easy to find $\frac{d^{2} g(t)}{d t^{2}}>0$, since $g(t)$ is strictly convex. Thus, by Jensen's inequality, we have $E(g(\bar{x}))>g(E(\bar{x}))$.

Finally, since $E(g(\bar{x}))=g(\mu)=g\left(\frac{(1+\theta)^{2}+1}{(1+\theta)^{2} \theta}\right)=\theta$, we obtain $E\left(\hat{\theta}_{M o M}\right)>\theta$ 
Theorem 3. The estimator $\hat{\theta}$ of $\theta$ is consistent and asymptotically normal:

$$
\sqrt{n}(\hat{\theta}-\theta) \stackrel{P}{\rightarrow} N\left(0, \frac{1}{\sigma^{2}}\right)
$$

The large-sample $100(1-\alpha) \%$ confidence interval for $\theta$ is given by:

$$
\hat{\theta} \pm z \frac{\alpha}{2} \frac{1}{\sqrt{n \sigma^{2}}}
$$

The proof is omitted because it is very similar to the proof of Theorem 4 [7]

\section{THE QUANTILE FUNCTION OF XLD}

It may be noted that $\mathrm{F}_{\mathrm{X}}(\mathrm{x})$ in equation (5) is continues and strictly increasing, so we for the quantile function of $\mathrm{X}$ is defined:

$$
\mathrm{Q}_{X}(u)=x_{u}=F_{X}^{-1}(u)
$$

For $u=F_{X L}(x)$, we give an explicit expression for $\mathrm{Q}_{X}(u)$ in terms of the Lambert $\mathrm{W}$ function in the following theorem and results.

Theorem 4. For any $\theta>0$, the $\mathrm{Q}_{X}(u)$ of the $\mathrm{XLD} X$ is:

$$
\mathrm{Q}_{X}(u)=x_{u}=-\frac{(1+\theta)^{2}}{\theta}-\frac{1}{\theta} W_{-1}\left[\frac{(1+\theta)^{2}}{\exp (1+\theta)^{2}}(u-1)\right], \mathrm{u} \in[0,1]
$$

Where $W_{-1}$ is the negative branch.

Proof. For any $\theta>0$ let $0 \prec u \prec 1$. From equation (5) we will solve the equation $u=F_{X L}(x)$ with respect to $\mathrm{x}$, by following the steps bellow:

$$
\begin{gathered}
\left(1+\frac{\theta x}{(1+\theta)^{2}}\right) e^{-\theta x}=(1-u) \\
{\left[(1+\theta)^{2}+\theta x\right] e^{-\theta x}=(1-u)(1+\theta)^{2}}
\end{gathered}
$$

We multiplying the both sides by $\left[-\exp (-1-\theta)^{2}\right]$ of the equation $(14)$, we get:

$$
-\left[(1+\theta)^{2}+\theta x\right] e^{-\left[(1+\theta)^{2}+\theta x\right]}=(u-1)(1+\theta)^{2} e^{-(1+\theta)^{2}}
$$

By using the definition of Lambert W function $(W(z) \exp (W(z))=z)$ [see Jodrá [13] for more details], we observe that $-\left[(1+\theta)^{2}+\theta x\right.$ ] is the Lambert $\mathrm{W}$ function of the real argument $(u-1)(1+\theta)^{2} e^{-(1+\theta)^{2}}$.

So, we have

$$
\begin{aligned}
W\left[(u-1)(1+\theta)^{2} e^{-(1+\theta)^{2}}\right] & =-\left[(1+\theta)^{2}+\theta x\right] \\
W\left[\frac{(1+\theta)^{2}}{e^{(1+\theta)^{2}}}(u-1) e^{-(1+\theta)^{2}}\right] & =-\left[(1+\theta)^{2}+\theta x\right]
\end{aligned}
$$

In addition to that, for any $\theta>0$ and $x>00$ it's obviously that $(1+\theta)^{2}+\theta x>0$ and it also checked that $(u-1)(1+\theta)^{2} e^{-(1+\theta)^{2}} \in\left(\frac{1}{e}, 0\right)$ since $0 \prec u \prec 1$ Thus, by taking into account the properties of the negative branch $W_{-1}$ of the Lambert $\mathrm{W}$ function, so the equation above (16) become:

$$
W_{-1}\left[\frac{(1+\theta)^{2}}{e^{(1+\theta)^{2}}}(u-1) e^{-(1+\theta)^{2}}\right]=-\left[(1+\theta)^{2}+\theta x\right]
$$

This in turn means the result that given before in Theorem 4 is complete. 


\section{SIMULATION}

We can see that the equation $\mathrm{F}(\mathrm{x})=\mathrm{u}$, where $\mathrm{u}$ is an observation from the uniform distribution on $(0 ; 1)$, can be solved explicitly in $\mathrm{x}$ ( we're going to use Lambert $\mathrm{W}$ function, because in this case $\mathrm{k}=1$ ).

In this subsection, we investigate the behavior of the ML estimators for a finite sample size (n). A simulation study consisting of the following steps is being carried out $\mathrm{N}=10000$ times for selected values of $(\theta ; \mathrm{n})$, where $\theta=0.1 ; 0.5 ; 1 ; 3 ; 5$ and $\mathrm{n}=20 ; 40 ; 100$

- Generate $U_{i}$ Uniform $(0 ; 1), i=1 \ldots . . . n$

- Generate $Y_{i}$ exponential $(\theta), i=1 \ldots \ldots . n$

- Generate $Z_{i} \operatorname{LD}(\theta), i=1 \ldots \ldots . n$

- If $U_{i} \leq p(\theta)$, then set $X_{i}=Y_{i}$, otherwise, set $X_{i}=Z_{i}, i=1 \ldots \ldots n$

$$
\text { average bais }=\frac{1}{N} \sum_{i=1}^{N}(\hat{\theta}-\theta)
$$

and the average square error:

$$
\operatorname{MSE}(\theta)=\frac{1}{N} \sum_{i=1}^{N}(\hat{\theta}-\theta)^{2}
$$

The result of the simulation is presented in Tables 1 and 2 . The following observations are made from the simulation study:

- For some given value of $\theta$, the average of: bias of $\hat{\theta}$ and mean square error of $\hat{\theta}$ are decreases as sample size $\mathrm{n}$ increases

- The mean square error (MSE) gets higher and following a similar ways for larger value of $\theta$ as we mentioned before.

\section{APPLICATION AND GOODNESS OF FIT}

Now we have used data of survival times (in months) of 94 sierra leone individus infected with Ebola virus showing in Table 3, which we compare LD, Zeghdoudi (ZD), see (Messaadia and Zeghdoudi [14]), Exponential, Xgamma see (Sen et al. [15]) and XLD.

\section{CONCLUSION}

In this work, we present a one-parameter distribution called XLD which is mixture of two known distribution Exponential $(\theta)$ and $\operatorname{LD}(\theta)$ distributions. Its survival properties have been discussed: moments, skewness, kurtosis, hazard and rate function, stochastic ordering, quantile function, the maximum likelihood method and method of moments. The XLD is flexible and likely model in describing real life timeto-event data. Lot of properties and simulation are given which confirm the goodness of fit and it's better than exponential, LD, Xgamma and ZD distributions.

\begin{tabular}{|c|c|c|c|c|c|}
\hline Bias & $\theta=0.1$ & $\theta=0.5$ & $\theta=1.5$ & $\theta=3$ & $\theta=6$ \\
\hline$n=20$ & 0.0032 & 0.01067 & 0.0485 & 0.276 & 0.785 \\
\hline$n=40$ & 0.00183 & 0.0150 & 0.0135 & 0.126 & 0.1770 \\
\hline$n=100$ & 0.000321 & 0.00404 & 0.0147 & 0.0452 & 0.0598 \\
\hline
\end{tabular}

Table 1 Average bias of the estimator $\hat{\theta}$.

MSE, mean square error.

\begin{tabular}{|c|c|c|c|c|c|}
\hline MSE & $\theta=0.1$ & $\theta=0.5$ & $\theta=1$ & $\theta=3$ & $\theta=5$ \\
\hline$n=20$ & $1,0854.10^{-5}$ & 0.000113 & 0.00236 & 0.0765 & 0.6177 \\
\hline$n=40$ & $3,357.10^{-6}$ & 0.000225 & 0.000183 & 0.01599 & 0.03135 \\
\hline$n=100$ & $1,0334 \cdot 10^{-7}$ & $1,640.10^{-5}$ & 0.000217 & 0.00204 & 0.00358 \\
\hline
\end{tabular}

Table 2 The average square error of the estimator $\hat{\theta}$. 
Table 3 Comparison between LD, Xgamma, ZD, Exp and XLD.

\begin{tabular}{lcccccc}
\hline $\begin{array}{l}\text { Survival Time } \\
\mathbf{m = 3 . 1 7} \mathbf{~ s = 2 . 0 9 5}\end{array}$ & Obs freq & LD $\hat{\theta}=0.522$ & Xgamma $\hat{\theta}=0.689$ & ZD $\hat{\theta}=0.852$ & Exp $\hat{\theta}=0.315$ & XLD $\hat{\theta}=0.467$ \\
\hline$[\mathbf{0 , 2}]$ & 45 & 38.262 & 37.652 & 30.339 & 43.937 \\
{$[\mathbf{2 , 4}]$} & 22 & 28.164 & 27.197 & 37.27 & 23.4 & 41.028 \\
{$[\mathbf{4 , 6}]$} & 17 & 15.075 & 16.342 & 17.743 & 12.463 & 25.855 \\
{$[\mathbf{6 , 8}]$} & 7 & 7.1187 & 7.7769 & 6.1658 & 6.6375 & 3.5351 \\
{$[\mathbf{8 , 1 0}]$} & 3 & 3.1423 & 3.2015 & 1.828 & 94.984 \\
Total & 94 & 94 & 94 & $\mathbf{1 4 . 2 3 6}$ & $\mathbf{1 . 8 6 1 9}$ & 3.3409 \\
$\chi^{2}$ & & $\mathbf{2 . 7 8 9 9}$ & $\mathbf{3 . 2 0 4 0}$ & & $\mathbf{1 . 6 4 4 6}$ \\
\hline
\end{tabular}

LD, Lindley; XLD, XLindley distribution; ZD, Zeghdoudi.

\section{CONFLICTS OF INTEREST}

The authors declare they have no conflicts of interest.

\section{AUTHORS' CONTRIBUTIONS}

Study conception and design: H. Zeghdoudi; analysis and interpretation of results: S. Chouia; draft manuscript preparation: S. Chouia and H. Zeghdoudi. All authors reviewed the results and approved the final version of the manuscript.

\section{Funding Statement}

This research was funded by PRFU Program of Ministry of Higher Education and Scientific Research No. C00L03UN230120180014.

\section{ACKNOWLEDGMENTS}

The authors acknowledge editor in chief, Prof. Dr.Mohammad Ahsanullah and the referee, of this journal for the constant encouragement to finalize the paper.

\section{REFERENCES}

1. D.V. Lindley, J. R. Stat. Soc. Series B Stat. Methodol. 20 (1958), 102-107.

2. M. Sankaran, Biometrics. 26 (1970), 145-149.

3. A. Asgharzadeh, S. Hassan, L.E. Bakouch, J. Appl. Stat. 40 (2013), 1-18.

4. H. Zeghdoudi, S. Nedjar, Afr. Stat. 11 (2016), 923-932.

5. H. Zeghdoudi, L. Bouchahed, Stat. Transit. New Series. 19 (2018), 61-74.

6. A. Beghriche, H. Zeghdoudi, Thail. Stat. 17 (2019), 179-189.

7. M.E. Ghitany, D.K. Al-Mutairi, S. Nadarajah, Math. Comput. Simul. 79 (2008), 279-287.

8. M.E. Ghitany, B. Atieh, S. Nadarajah, Math. Comput. Simul. 78 (2008), 493-506.

9. H. Zeghdoudi, S. Nedjar, J. Appl. Prob. Stat. 11 (2016), 129-138.

10. H. Zeghdoudi, S. Nedjar, J. Comp. Appl. Math. 298 (2016), 167-174.

11. R. Shanker, S. Sharma, Appl. Math. 4 (2013), 363-368.

12. R.E. Glaser, J. Amer. Statist. Assoc. 75 (1980), 667-672.

13. P. Jodrá, J. Math. Comput. Simul. 81 (2010), 851-859.

14. H. Messaadia, H. Zeghdoudi, Int. J. Comput. Sci. Math. 9 (2018), 58-65.

15. S. Sen, S. Maiti, N. Chandra, J. Mod. Appl. Stat. Methods. 15 (2016), 38. 